\title{
Robust Frequency Domain ARMA Modelling
}

\author{
Jonas Gillberg, Fredrik Gustafsson, Rik Pintelon \\ Division of Automatic Control \\ Department of Electrical Engineering \\ Linköpings universitet, SE-581 83 Linköping, Sweden \\ WWW: http://WwW. control.isy.liu.se \\ E-mail: gillberg@isy.liu.se, gustafsson@isy.liu.se, \\ Rik.Pintelon@vub.ac.be
}

5th November 2004

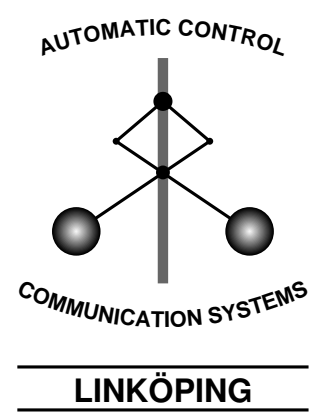

Report no.: LiTH-ISY-R-2694

Submitted to 14th IFAC Symposium on System Identification, SYSID-2006

Technical reports from the Control \& Communication group in Linköping are available at http://www.control.isy.liu.se/publications. 


\begin{abstract}
In this paper a method for the rejection of frequency domain outliers is proposed. The algorithm is based on the work by Huber on M-estimators and the concept of influence function introduced by Hampel. The estimation takes place in the context of frequency domain continuous-time ARMA modelling, but the method can be also be applied to the discrete time case.
\end{abstract}

Keywords: robust estimation, outlier rejection, continuous-time, ARMA, CARMA, frequency Domain, influence function, M-estimator 


\title{
Robust Frequency Domain ARMA Modelling
}

\author{
Jonas Gillberg, Fredrik Gustafsson, Rik Pintelon
}

2005-09-05

\begin{abstract}
In this paper a method for the rejection of frequency domain outliers is proposed. The algorithm is based on the work by Huber on M-estimators and the concept of influence function introduced by Hampel. The estimation takes place in the context of frequency domain continuous-time ARMA modelling, but the method can be also be applied to the discrete time case.
\end{abstract}

\section{Introduction}

A characteristic industrial signal processing problem is vibration analysis using tachometer measurements on rotating axles. Motivated by recent advances in system identification in the frequency domain (Pintelon and Schoukens, 2001; Ljung, 1999), a frequency domain approach for such analysis was outlined in an earlier paper (Gillberg and Gustafsson, 2005) by the authors and compared at a theoretical level to the time domain algorithm proposed in (Persson, 2002; Persson and Gustafsson, 2001). Among other things, the new method involved vibrational modelling using a continuous-time AR model and the restriction of the frequency interval to be used in the estimation. Interpolation was also performed in order to counter the effect of non-uniform sampling.

In the same paper the main specifications on a procedure aimed at highsensitivity vibration analysis were listed:

1. Being based on parametric physical models of the vibration.

2. Operate on short data batches in a prespecified speed interval where the data pass several quality checks.

3. Potential to reject wide band disturbances that are non-interfering with the vibration.

4. Potential to reject narrow band disturbances that are interfering with the vibration.

The methods given in (Persson, 2002; Persson and Gustafsson, 2001) and (Gillberg and Gustafsson, 2005) successfully solves the first three specifications, but not the last one.

The problem of narrow-band disturbances occurs in several applications. For instance in an automotive drive-line, see Figure 1, where the vibrations can be due to engine knock or gear shifts. In robotics there are vibrations from the load, just to mention a few. In this paper the focus will therefore be on robustness against these narrow band disturbances. 


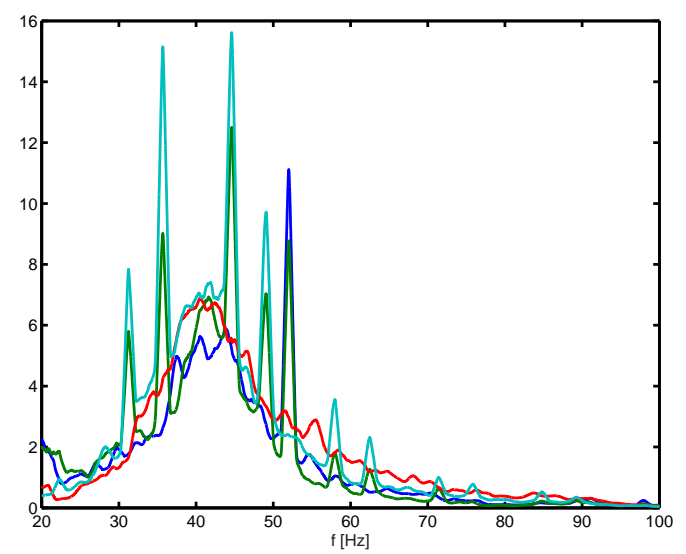

Figure 1: Smoothed spectrum for vibrations extracted from ABS system signals

\section{Outline}

The outline of the paper will be the following. First in Section 3 the frequency domain continuous-time ARMA (CARMA) modelling and identification approach will be described. Then in Section 4 the identification criterion is modified in order to facilitate rejection of frequency domain outliers. The concept of M-estimators and the influence function are also introduced. In Section 5 the asymptotic variance and gross sensitivity of the estimator are calculated, and measures are taken in order to assure consistency in the case of correct modelling. A certain choice of criterion is also proved to make an optimal tradeoff between decreased bias due to outliers and increased variance due to a more insensitive method. Finally in Section 6 the algorithm is illustrated by a numerical example.

\section{Frequency Domain CARMA Estimation}

In this paper we shall consider continuous-time ARMA models represented as

$$
y(t)=G_{c}(p) e(t)
$$

where $e(t)$ is continuous time white noise such that

$$
\begin{aligned}
E[e(t)] & =0 \\
E[e(t) e(s)] & =\sigma^{2} \delta(t-s)
\end{aligned}
$$

The operator $p$ is here the differentiation operator. We assume that $G(p)$ is strictly proper, so $y(t)$ itself does not have a white-noise component, but is a well defined second order, stationary process. Its spectrum (spectral density) can be written as

$$
\Phi_{c}(\omega)=\sigma^{2}\left|G_{c}(i \omega)\right|^{2}
$$

We shall consider a general model parameterization

$$
G_{c}(p, \theta)
$$


where the model parameter vector $\theta$ includes the noise variance $\lambda$ (whose true value is $\sigma^{2}$ ). The transfer function $G$ can be parameterized by $\theta$ in an arbitrary way, for example by the conventional numerator and denominator parameters:

$$
\begin{aligned}
& G(p, \theta)=\frac{B(p)}{A(p)} \\
& A(p)=p^{n}+a_{1} p^{n-1}+a_{2} p^{n-2}+\cdots+a_{n} \\
& B(p)=p^{m}+b_{1} p^{m-1}+\cdots+b_{m} \\
& \theta=\left[\begin{array}{lllllllll}
a_{1} & a_{2} & \ldots & a_{n} & b_{1} & b_{2} & \ldots & b_{m} & \lambda
\end{array}\right]^{T} .
\end{aligned}
$$

Let us define the truncated Fourier transformation of the continuous time output $\{y(t): t \in[0, T]\}$ in expression (1) as

$$
Y_{T}(i \omega)=\frac{1}{\sqrt{T}} \int_{0}^{T} y(t) e^{-i \omega t} d t .
$$

A complicating element in a practical estimation procedure is that we do not have access to the entire continuous time realization of the output. Instead we have a finite number of samples of $y(t)$ at time instances $\left\{t_{1}, t_{2}, \ldots, t_{N}\right\}$. This is an important issue which have been dealt with in the case of equidistant sampling in a previous paper by the first author (Gillberg and Ljung, 2005b). The case of non-uniform sampling we refer the reader to a technical reports associated with two other papers submitted to this conference (Gillberg and Ljung, 2005a) (Gillberg and Ljung, 2004a).

However, if we have an estimate of the continuous-time Fourier transform, the continuous-time periodogram is

$$
\hat{\hat{\Phi}}\left(i \omega_{n}\right)=\left|Y_{T}\left(i \omega_{n}\right)\right|^{2} \in \operatorname{Exp} \Phi\left(\omega_{n}, \theta_{0}\right)
$$

and the transform will be asymptotically independent at the frequencies $\omega_{k}, k=$ $1, \ldots, N_{\omega}$ where $\omega_{k}=2 \pi k / T, k \in \mathcal{N}$. In this paper we will focus treat the continuous-time periodogram estimates as our "measurements" and will consider them to be completely uncorrelated.

When an estimate of the power spectrum is available a model can be identified by the following Maximum-Likelihood (ML) procedure described in (Gillberg and Ljung, 2004b) and (Gillberg, 2004)

$$
\hat{\theta}=\arg \min _{\theta} \sum_{k=1}^{N_{\omega}} \frac{\hat{\hat{\Phi}}\left(i \omega_{k}\right)}{\Phi\left(i \omega_{k}, \theta\right)}+\log \Phi\left(i \omega_{k}, \theta\right) .
$$

where the asymptotic Fisher information at the true parameters $\theta_{0}$ is

$$
J\left(\theta_{0}\right)=\sum_{n=1}^{N_{\omega}} \frac{\Phi_{\theta_{0}}^{\prime}\left(\omega_{n}, \theta_{0}\right)}{\Phi\left(\omega_{n}, \theta_{0}\right)} \frac{\Phi_{\theta}^{\prime}\left(\omega_{n}, \theta_{0}\right)^{T}}{\Phi\left(\omega_{n}, \theta_{0}\right)} .
$$

This approach forms the basis for the work presented in the remaining part of the paper.

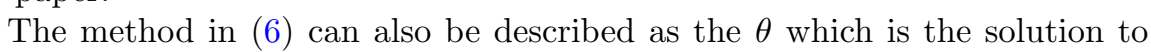
the vector equation

$$
\sum_{n=1}^{N_{\omega}} \frac{\Phi_{\theta}^{\prime}\left(\omega_{n}, \theta\right)}{\Phi\left(\omega_{n}, \theta\right)}\left(\frac{\hat{\hat{\Phi}}\left(\omega_{n}\right)}{\Phi\left(\omega_{n}, \theta\right)}-1\right)=0 .
$$

where $\Phi_{\theta}^{\prime}\left(\omega_{n}, \theta\right)$ is the gradient of the spectrum with respect to the parameters. 


\section{M-estimators and Outlier Rejection}

If the model is the correct one, the maximum-likelihood method is known to be the best possible (optimal) estimator with consistency and asymptotic efficiency (Wald, 1949)(Cramér, 1046). If there, on the other hand, are unmodelled outliers present, the method might not be optimal. This may lead us to consider robust estimators which give up efficiency at the true model in exchange for reasonable performance if the model is not the true(Casella and Berger, 2002). Therefore we introduce the following method

$$
\Psi(\hat{\hat{\Phi}}, \theta)=\sum_{n=1}^{N_{\omega}} \frac{\Phi_{\theta}^{\prime}\left(\omega_{n}, \theta\right)}{\Phi\left(\omega_{n}, \theta\right)} \psi\left(\frac{\hat{\hat{\Phi}}\left(\omega_{n}\right)}{\Phi\left(\omega_{n}, \theta\right)}\right)=0 .
$$

inspired by the M-estimators introduced by Huber (Huber, 1981). The unknown here is the function $\psi$ which will be found using the influence function approach introduced by Hampel (Hampel et al., 1986). Certain measures must however be taken in order to assure that the estimates are consistent when periodogram originate from the assumed model in (5). This implies that

$$
\begin{aligned}
\int \psi\left(\frac{\hat{\hat{\Phi}}\left(i \omega_{n}\right)}{\Phi\left(i \omega_{n}, \theta_{0}\right)}\right) & d F_{n}\left(\hat{\hat{\Phi}}\left(i \omega_{n}\right)\right)= \\
\int \psi(x) d G(x) & =0
\end{aligned}
$$

where we from now on define the distributions

$$
\begin{aligned}
F_{n} & \sim \operatorname{Exp} \Phi\left(i \omega_{n}, \theta_{0}\right) \\
G & \sim \operatorname{Exp} 1
\end{aligned}
$$

In this context we define the solution to equation (맘 as a vector valued stochastic variable $T(F)$ with a distribution dependent on $F=\left(F_{1}, \ldots, F_{N_{\omega}}\right)$, the distribution of the periodogram $\hat{\hat{\Phi}}\left(i \omega_{n}\right)$. The so called influence function introduced by Hampel is then defined as

$$
I F(\hat{\hat{\Phi}} ; T, F)=\lim _{h \rightarrow 0} \frac{T\left((1-h) F+h \delta_{\hat{\hat{\Phi}}}\right)-T(F)}{h}
$$

and measures the asymptotic bias caused by contamination in the data. Here $\delta_{\hat{\hat{\Phi}}}$ is the multi-variable measure which puts the mass 1 at $\hat{\hat{\Phi}}=\left(\hat{\hat{\Phi}}\left(i \omega_{1}\right), \ldots, \hat{\hat{\Phi}}\left(\omega_{N_{\omega}}\right)\right.$.

\section{Influence Function and Asymptotic Variance}

In the case of the estimator in (8) it is possible to show that (see p.101 in (Hampel et al., 1986))

$$
I F(\hat{\hat{\Phi}} ; T, F)=M(\Psi, T(F))^{-1} \Psi(\hat{\hat{\Phi}}, T(F))
$$

where

$$
M(\Psi, F)=-\int(\Psi(\hat{\hat{\Phi}}, \theta))_{\theta=T(F)}^{\prime} d F(\hat{\hat{\Phi}})
$$


It is also possible to show (see p.85 in (Hampel et al., 1986)) that the asymptotic variance of the parameter estimates will be

$$
\begin{aligned}
V(T, F) & =\int I F(\hat{\hat{\Phi}} ; T, F) I F(\hat{\hat{\Phi}} ; T, F) d F(\hat{\hat{\Phi}}) \\
& =M(\psi, F)^{-1} Q(\psi, F) M(\psi, F)^{-T}
\end{aligned}
$$

where

$$
Q(\Psi, F)=\int \Psi(\hat{\hat{\Phi}}, T(F)) \Psi^{T}(\hat{\hat{\Phi}}, T(F)) d F(\hat{\hat{\Phi}})
$$

Here

$$
\begin{array}{r}
M(\Psi, F)=-\int(\Psi(\hat{\hat{\Phi}}, \theta))_{\theta=\theta_{0}}^{\prime} d F(\hat{\hat{\Phi}}) \\
=-\sum_{n=1}^{N_{\omega}} \int\left(\frac{\Phi_{\theta}^{\prime}\left(\omega_{n}, \theta\right)}{\Phi\left(\omega_{n}, \theta\right)} \psi\left(\frac{\hat{\hat{\Phi}}\left(\omega_{n}\right)}{\Phi\left(\omega_{n}, \theta\right)}\right)\right)_{\theta=\theta_{0}}^{\prime} d F(\hat{\hat{\Phi}})
\end{array}
$$

and

$$
\begin{array}{r}
-\left(\frac{\Phi_{\theta}^{\prime}\left(\omega_{n}, \theta\right)}{\Phi\left(\omega_{n}, \theta\right)} \psi\left(\frac{\hat{\hat{\Phi}}\left(\omega_{n}\right)}{\Phi\left(\omega_{n}, \theta\right)}\right)\right)_{\theta=\theta_{0}}^{\prime}= \\
\left(\frac{\Phi_{\theta}^{\prime}\left(\omega_{n}, \theta_{0}\right)}{\Phi\left(\omega_{n}, \theta_{0}\right)} \frac{\Phi_{\theta}^{\prime}\left(\omega_{n}, \theta_{0}\right)^{T}}{\Phi\left(\omega_{n}, \theta_{0}\right)}-\frac{\Phi_{\theta}^{\prime \prime}\left(\omega_{n}, \theta_{0}\right)}{\Phi\left(\omega_{n}, \theta_{0}\right)}\right) \psi\left(\frac{\hat{\hat{\Phi}}\left(\omega_{n}\right)}{\Phi\left(\omega_{n}, \theta_{0}\right)}\right) \\
+\frac{\Phi_{\theta}^{\prime}\left(\omega_{n}, \theta_{0}\right)}{\Phi\left(\omega_{n}, \theta_{0}\right)} \frac{\Phi_{\theta}^{\prime}\left(\omega_{n}, \theta_{0}\right)^{T}}{\Phi\left(\omega_{n}, \theta_{0}\right)} \frac{\hat{\hat{\Phi}}\left(\omega_{n}\right)}{\Phi\left(\omega_{n}, \theta_{0}\right)} \psi^{\prime}\left(\frac{\hat{\hat{\Phi}}\left(\omega_{n}\right)}{\Phi\left(\omega_{n}, \theta_{0}\right)}\right) .
\end{array}
$$

Because of (9) and (10) the expected value of first expression on the right hand side of the equation above will be zero. Therefore we will have

$$
M(\Psi, F)=J(\theta) \int x \psi^{\prime}(x) d G(x) .
$$

Since the periodogram at different frequencies are assumed independent we also have

$$
\begin{aligned}
Q(\Psi, F) & =\int \Psi(\hat{\hat{\Phi}}, T(F)) \Psi^{T}(\hat{\hat{\Phi}}, T(F)) d F(\hat{\hat{\Phi}}) \\
& =J(\theta) \int \psi^{2}(x) d G(x)
\end{aligned}
$$

where $J$ is the Fischer information in (7) and the asymptotic variance will be

$$
V(T, F)=\frac{\int \psi^{2}(x) d G(x)}{\left(\int x \psi^{\prime}(x) d G(x)\right)^{2}} J^{-1}(\theta)
$$




\subsection{Optimal B-Robust Estimator}

Assume now that we want to find a function $\psi$ that limits the gross sensitivity measured as

$$
\gamma_{u}^{*}(T, F)=\sup _{\hat{\hat{\Phi}}}\{|I F(\hat{\hat{\Phi}} ; T, F)|\}
$$

where |.| means taking the absolute value of each vector component, while at the same time minimizes the trace of the variance $\operatorname{Tr} V(T, F)$. In our case we will have

$$
\begin{aligned}
\operatorname{Tr} V(T, F) & =\operatorname{Tr} \frac{\int \psi^{2}(x) d G(x)}{\left(\int x \psi^{\prime}(x) d G(x)\right)^{2}} J^{-1}(\theta) \\
& =\frac{\int \psi^{2}(x) d G(x)}{\left(\int x \psi^{\prime}(x) d G(x)\right)^{2}} \operatorname{Tr} J^{-1}(\theta)
\end{aligned}
$$

and

$$
\begin{array}{r}
|I F(\hat{\hat{\Phi}} ; T, F)|=\left|M(\Psi, T(G))^{-1} \Psi(\hat{\hat{\Phi}}, T(F))\right| \\
=\left|\frac{J(\theta)^{-1}}{\int x \psi^{\prime}(x) d G(x)} \sum_{n=1}^{N_{\omega}} \frac{\Phi_{\theta}^{\prime}\left(\omega_{n}, \theta\right)}{\Phi\left(\omega_{n}, \theta\right)} \psi\left(\frac{\hat{\hat{\Phi}}\left(\omega_{n}\right)}{\Phi\left(\omega_{n}, \theta\right)}\right)\right| \\
\leq\left|\frac{b}{\int x \psi^{\prime}(x) d G(x)}\right| \sum_{n=1}^{N_{\omega}}\left|J(\theta)^{-1} \frac{\Phi_{\theta}^{\prime}\left(\omega_{n}, \theta\right)}{\Phi\left(\omega_{n}, \theta\right)}\right|
\end{array}
$$

According to Theorem 1 and Equation (2.4.10) on p. 122 in (Hampel et al., 1986) a version of the so called Huber function

$$
\begin{aligned}
\psi(x) & =[x-1-a]_{-b}^{b} \\
& = \begin{cases}b & \text { if } b<x-1-a \\
x-1-a & \text { if }-b<x-1-a \leq b \\
-b & \text { if } x-1-a \leq-b\end{cases}
\end{aligned}
$$

minimizes

$$
\frac{\int \psi^{2}(x) d G(x)}{\left(\int x \psi^{\prime}(x) d G(x)\right)^{2}}
$$

among all mappings $\psi$ that satisfy

$$
\begin{gathered}
\int \psi(x) d G(x)=0 \\
\sup _{x}\left|\frac{\psi(x)}{\int x \psi^{\prime}(x) d G(x)}\right| \leq \frac{b}{\left|\int x \psi^{\prime}(x) d G(x)\right|}
\end{gathered}
$$

Therefore, using the function in (14) in

$$
\sum_{n=1}^{N_{\omega}} \frac{\Phi_{\theta}^{\prime}\left(\omega_{n}, \theta\right)}{\Phi\left(\omega_{n}, \theta\right)} \psi\left(\frac{\hat{\hat{\Phi}}\left(\omega_{n}\right)}{\Phi\left(\omega_{n}, \theta\right)}\right)=0
$$


will minimize

$$
\operatorname{Tr} V(T, F)=\frac{\int \psi^{2}(x) d G(x)}{\left(\int x \psi^{\prime}(x) d G(x)\right)^{2}} \operatorname{Tr} J^{-1}(\theta)
$$

while

$$
\sup _{\hat{\hat{\Phi}}}|I F(\hat{\hat{\Phi}} ; T, F)| \leq b \frac{\sum_{n=1}^{N_{\omega}}\left|J(\theta)^{-1} \frac{\Phi_{\theta}^{\prime}\left(\omega_{n}, \theta\right)}{\Phi\left(\omega_{n}, \theta\right)}\right|}{\left|\int x \psi^{\prime}(x) d G(x)\right|}
$$

\subsection{Computing a}

The parameter $b$ in (14) can be considered a tuning factor which is selected by the user in order to strike a balance between the asymptotic bias and variance of the parameter estimates. The factor $a$ is then selected such that the estimator is asymptotically unbiased when the data is produced by the model. That is

$$
\int \psi(x) d G(x)=\int_{0}^{\infty}[x-1-a]_{-b}^{b} e^{-x} d x=0 .
$$

This means that three different cases have to be considered.

\subsection{Case 1}

The first, when $b \leq-1-a$ which is trivial since

$$
\int_{0}^{\infty}[x-1-a]_{-b}^{b} e^{-x} d x=b=0
$$

yields $b=0$.

\subsection{Case 2}

The second case is when $-b<-1-a \leq b$. Here

$$
\begin{aligned}
\int_{0}^{\infty}[x-1-a]_{-b}^{b} e^{-x} d x= & \int_{0}^{1+a+b}(x-1-a) e^{-x} d x \\
& +\int_{1+a+b}^{\infty}(x-1-a) e^{-x} d x .
\end{aligned}
$$

The first integral in this expression will be

$$
\begin{aligned}
& \int_{0}^{1+a+b}(x-1-a) e^{-x} d x= \\
& {\left[-e^{-x}-x e^{-x}+(1+a)\right]_{0}^{1+a+b}} \\
& =\left[(a-x) e^{-x}\right]_{0}^{1+a+b} \\
& =(-1-b) e^{-b} e^{-(1+a)}-a
\end{aligned}
$$

The second integral is

$$
\begin{aligned}
& \int_{1+a+b}^{\infty} b e^{-x} d x \\
& =\left[-b e^{-x}\right]_{1+a+b}^{\infty}=b e^{-b} e^{-(1+a)}
\end{aligned}
$$


This means that given $b$ we have to chose $a(b)$ such that

$$
\begin{array}{r}
(-1-b) e^{-b} e^{-(1+a)}-a+b e^{-b} e^{-(1+a)}= \\
-e^{-b} e^{-(1+a)}-a=0
\end{array}
$$

This means that in this case

$$
\left\{\begin{array}{l}
e^{-b} e^{-(1+a)}+a=0 \\
1+a \leq b
\end{array}\right.
$$

\subsection{Case 3}

In the third case we have $-1-a \leq-b$ and

$$
\begin{aligned}
& \int_{0}^{\infty}[x-1-a]_{-b}^{b} e^{-x} d x=-\int_{0}^{1+a-b} b e^{-x} d x \\
& \int_{1+a-b}^{1+a+b}(x-1-a) e^{-x} d x+\int_{1+a+b}^{\infty}(x-1-a) e^{-x} d x
\end{aligned}
$$

The first integral will in this case be

$$
\begin{aligned}
-\int_{0}^{1+a-b} b e^{-x} d x & =\left[b e^{-x}\right]_{0}^{1+a-b} \\
= & b e^{b} e^{-(1+a)}-b
\end{aligned}
$$

while the second is

$$
\begin{aligned}
& \int_{1+a-b}^{1+a+b}(x-1-a) e^{-x} d x= \\
& =\left[(a-x) e^{-x}\right]_{1+a-b}^{1+a+b} \\
& =(a-1-a-b) e^{-b} e^{-(1+a)}-(a-1-a+b) e^{b} e^{-(1+a)} \\
& =\left((-b-1) e^{-b}-(b-1) e^{b}\right) e^{-(1+a)}
\end{aligned}
$$

The third integral is the same as previously

$$
\int_{1+a+b}^{\infty}(x-1-a) e^{-x}=b e^{-b} e^{-(1+a)}
$$

This means that we have

$$
\begin{aligned}
& \int_{0}^{\infty}[x-1-a]_{-b}^{b} e^{-x} d x= \\
& b e^{b} e^{-(1+a)}-b+\left((-b-1) e^{-b}-(b-1) e^{b}\right) e^{-(1+a)} \\
& +b e^{-b} e^{-(1+a)} \\
& =\left(e^{b}-e^{-b}\right) e^{-(1+a)}-b=0
\end{aligned}
$$

and the solution is

$$
\left\{\begin{array}{l}
a=\ln \frac{e^{b}-e^{-b}}{b}-1 \\
1+a \geq b
\end{array}\right.
$$




\subsection{Equations}

This means that $a(b)$ is defined by the equations

$$
\begin{cases}e^{-b} e^{-(1+a)}+a=0 & \text { if } 1+a \leq b \\ e^{1+a}=\ln \frac{e^{b}-e^{-b}}{b} & \text { if } 1+a \geq b\end{cases}
$$

In Figure 2 the parameter $a$ is presented as a function of the parameter $b$. The breakpoint between the two sets of solutions occurs when $b \approx 0.7968$.

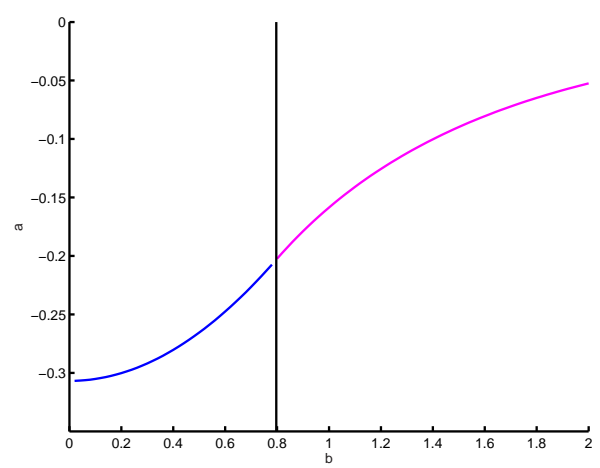

Figure 2: The parameter $a$ as a function of the parameter $b$. The breakpoint between the two solution occurs at $b \approx 0.7968$

\section{Numerical Example}

In this section we will compare the efficiency and robustness of the proposed estimation method in (8) and (14) with that of the ML approach in (6). The objective is to estimate the parameters of the continuous-time spectrum

$$
\Phi(i \omega)=\frac{\lambda}{\left|(i \omega)^{2}+a_{1}(i \omega)+a_{2}\right|^{2}}
$$

where $a_{1}=3$ and $a_{2}=2$. The underlying continuous-time system is the autoregressive model

$$
y(t)=\frac{\sigma}{p^{2}+a_{1} p+a_{2}} e(t)
$$

where $e(t)$ is continuous-time Gaussian white noise.

In Figure 3 we have computed the standard deviation for the parameter estimates of the continuous-time model found in (18). Both the new and the original ML method have been used. Simulations have been performed using $N_{M C}=1000$ Monte-Carlo runs for each value of $b$. As expected the standard deviation of the robust method will increase as $b$ decrease, but it is interesting to note that it only approximately double for such a small value as $b=0.1$. In the estimation we have used frequencies $w=\{0: 0.01: 2 \pi\}$

In Figure 4 we have estimated the same parameters as in the example problem above. In order to simulate outliers, we have also introduced an additive, 


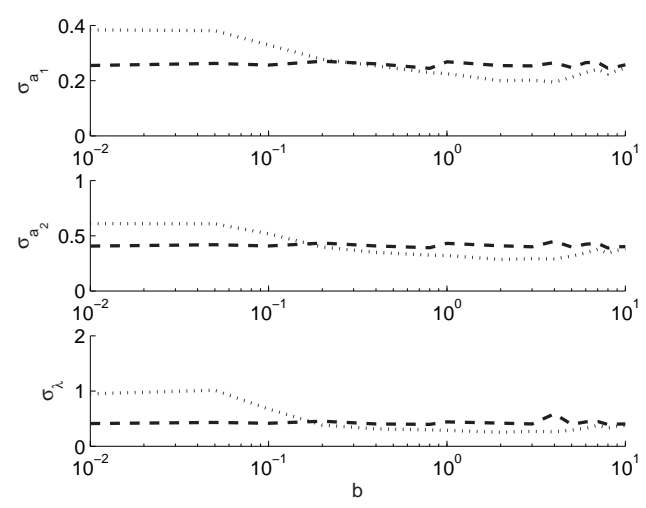

Figure 3: The standard deviations of the parameter estimates. Robust (dotted) and ML method (dashed).

random and exponentially distributed disturbance at $5 \%$ of all frequencies, with a variance which is twice the maximum value of the power spectrum in (17). From the figure one can see that the outliers cause bias in the param-

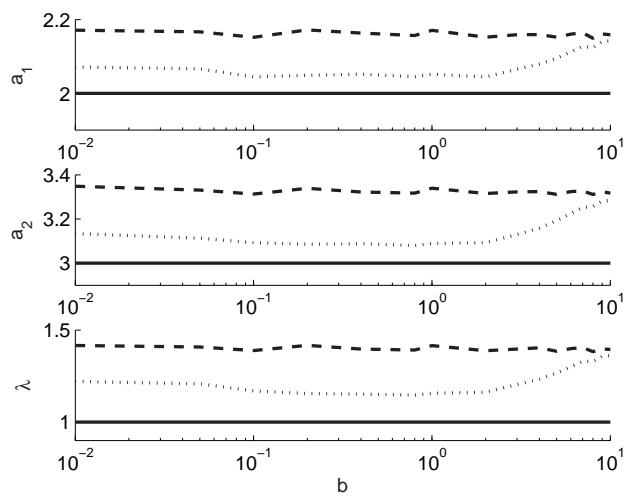

Figure 4: The bias for the parameter estimates. True (solid), ML method (dashed) and robust (dotted).

eter estimates when the ML method is utilized. This bias is then reduced by approximately $50 \%$ with the use of the more robust criterion.

\section{Conclusions}

In this paper a method for the rejection of frequency domain outliers is proposed. The algorithm is based on the work by Huber on M-estimators and the concept of influence function introduced by Hampel. The estimation takes place in the context of frequency domain continuous-time ARMA modelling, but the method can be also be applied to the discrete time case. It is also proved that a certain choice of criterion will produce an optimal tradeoff between bias and variance 
under certain assumptions. Finally the method is illustrated by a numerical example which shows that the bias can be reduced by approximately $50 \%$.

\section{References}

Casella, G. and R.L. Berger (2002). Statistical Inference. Duxbury. Pacific Grove, CA.

Cramér, H. (1046). Mathematical Methods of Statistics. Princeton University Press. Princeton, NJ.

Gillberg, J. (2004). Methods for frequency domain estimation of continuous-time models. Lic. Dissertation Linköping Studies in Science and Technology Thesis No. 1133. Department of Electrical Engineering, Linköping University. SE-581 83 Linköping, Sweden.

Gillberg, J. and F. Gustafsson (2005). Frequency-domain continuous-time AR modelling using non-uniformly sampled measurements. In: Proceedings of IEEE Int Conf on Acoustics, Speech and Signal Processing in Philadelphia, USA.

Gillberg, J. and L. Ljung (2004a). Frequency-domain identification of continuous-time ARMA models: Interpolation and non-uniform sampling. Technical Report LiTH-ISY-R-2625. Department of Electrical Engineering, Linköping University. SE-581 83 Linköping, Sweden.

Gillberg, J. and L. Ljung (2004b). Frequency-domain identification of continuous-time ARMA models: Interpolation and non-uniform sampling. Technical Report LiTH-ISY-R-2625. Department of Electrical Engineering, Linköping University. SE-581 83 Linköping, Sweden.

Gillberg, J. and L. Ljung (2005a). Frequency-domain identification of continuous-time ARMA models from non-uniformly sampled data. Technical Report LiTH-ISY-R-2693. Department of Electrical Engineering, Linköping University. SE-581 83 Linköping, Sweden.

Gillberg, J. and L. Ljung (2005b). Frequency-domain identification of continuous-time ARMA models from sampled data. In: Proc. 16th IFAC World Congress.

Hampel, F. R., E.M. Ronchetti, P.J. Rousseeuw and W.A. Stael (1986). Robust Statistics: the approach based on influence function. John Wiley \& Sons.

Huber, P. (1981). Robust Statistics. John Wiley \& Sons.

Ljung, L. (1999). System Identification: Theory for the User. Prentice-Hall. Upper Saddle River.

Persson, N. (2002). Event based sampling with applicaion to spectral estimation. Lic. Dissertation Linköping Studies in Science and Technology Thesis No. 981. Department of Electrical Engineering, Linköping University. SE-581 83 Linköping, Sweden. 
Persson, N. and F. Gustafsson (2001). Event based sampling with application to vibration analysis in pneumatic tires. In: Proceedings of IEEE Int Conf on Acoustics, Speech and Signal Processing in Salt Lake City, USA.

Pintelon, R. and J. Schoukens (2001). System Identification - A Frequency Domain Approach. IEEE Press. Piscataway, NJ.

Wald, A. (1949). Note on the consistency of the maximum likelihood estimate. Anti. Math. Statis. 20, 595-601. 


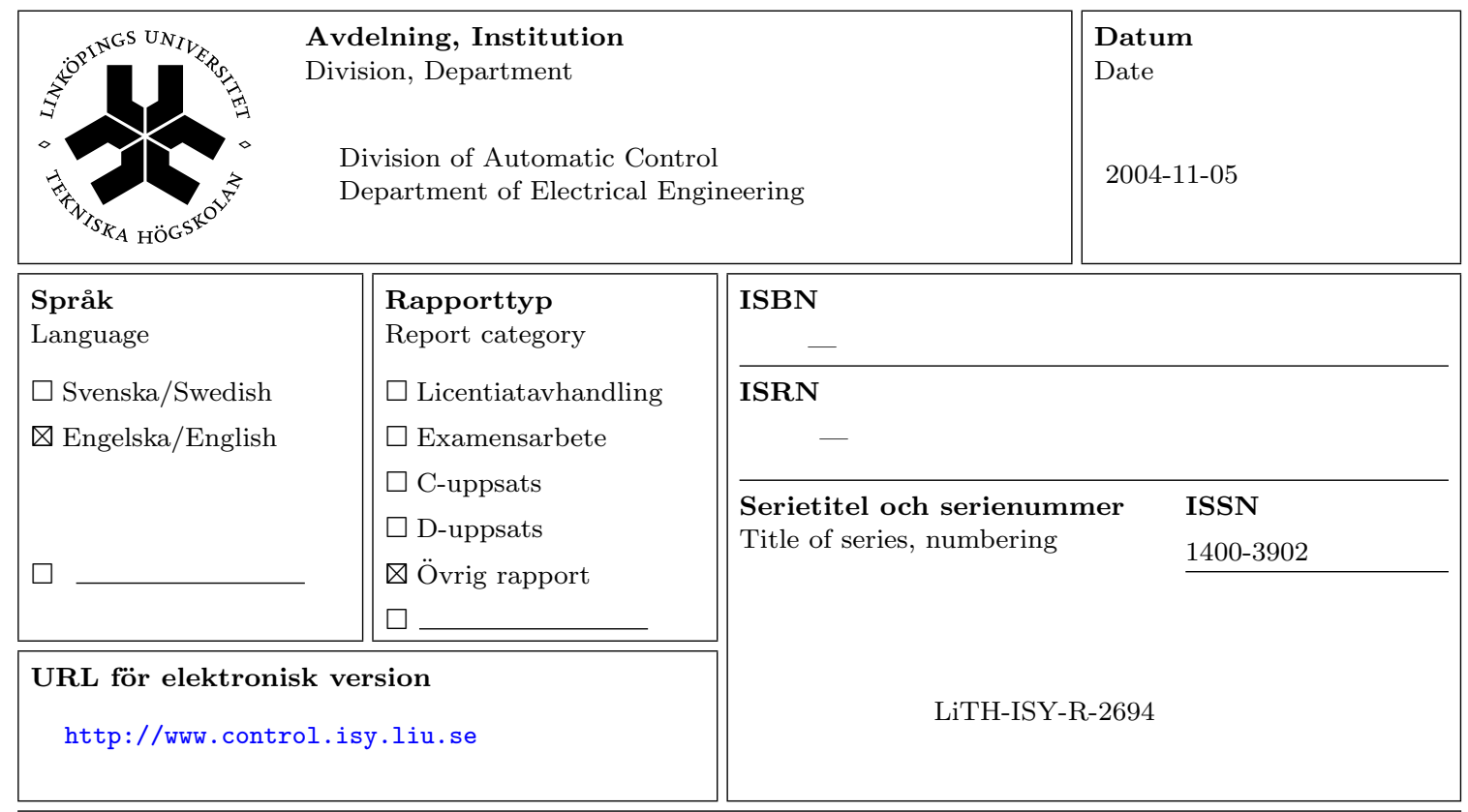

Titel Robust Frequency Domain ARMA Modelling

Title

Författare Jonas Gillberg, Fredrik Gustafsson, Rik Pintelon

Author

Sammanfattning

Abstract

In this paper a method for the rejection of frequency domain outliers is proposed. The algorithm is based on the work by Huber on M-estimators and the concept of influence function introduced by Hampel. The estimation takes place in the context of frequency domain continuous-time ARMA modelling, but the method can be also be applied to the discrete time case. 